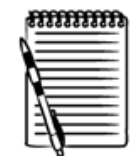

PRESS

RELEASE
- Additional materials are published online only. To view these files please visit the journal online (http://thorax.bmj. com/content/67/4.toc).

${ }^{1}$ Imperial College School of Medicine, Imperial College, London, UK

${ }^{2}$ NHLI Respiratory Sciences, Imperial College, London, UK ${ }^{3}$ Department of Haematology, Hammersmith Hospital, Imperial College Healthcare NHS Trust, London, UK

${ }^{4}$ Department of Clinical Biochemistry, Hammersmith Hospital, Imperial College Healthcare NHS Trust, London, UK

${ }^{5}$ Department of Imaging, Hammersmith Hospital, Imperial College Healthcare NHS Trust, London, UK

${ }^{6}$ School of Computing Sciences, University of East Anglia, UK

${ }^{7}$ Investigative Sciences, Imperial College, London, UK

${ }^{8} \mathrm{NHLI}$ Cardiovascular Sciences, Imperial College, London, UK ${ }^{9}$ Department of Respiratory Medicine, Hammersmith Hospital, Imperial College Healthcare NHS Trust, London, UK

\section{Correspondence to}

Claire L Shovlin, Respiratory Medicine, Hammersmith Hospital, Du Cane Rd, London W12 ONN, UK;

c.shovlin@imperial.ac.uk

Received 7 September 2011 Accepted 17 November 2011 Published Online First 14 December 2011

\title{
Low serum iron levels are associated with elevated plasma levels of coagulation factor VIII and pulmonary emboli/deep venous thromboses in replicate cohorts of patients with hereditary haemorrhagic telangiectasia
}

\author{
John A Livesey, ${ }^{1,2}$ Richard A Manning, ${ }^{3}$ John H Meek, ${ }^{4}$ James E Jackson, ${ }^{5}$ \\ Elena Kulinskaya, ${ }^{6}$ Michael A Laffan,, ${ }^{3,7}$ Claire L Shovlin ${ }^{8,9}$
}

\begin{abstract}
Background Elevated plasma levels of coagulation factor VIII are a strong risk factor for pulmonary emboli and deep venous thromboses.

Objectives To identify reversible biomarkers associated with high factor VIII and assess potential significance in a specific at-risk population.

Patients/Methods 609 patients with hereditary haemorrhagic telangiectasia were recruited prospectively in two separate series at a single centre. Associations between log-transformed factor VIII measured 6 months from any known thrombosis/illness, and patient-specific variables including markers of inflammation and iron deficiency, were assessed in stepwise multiple regression analyses. Age-specific incidence rates of radiologically proven pulmonary emboli/deep venous thromboses were calculated, and logistic regression analyses performed.
\end{abstract}

Results In each series, there was an inverse association between factor VIII and serum iron that persisted after adjustment for age, inflammation and/or von Willebrand factor. Iron response elements within untranslated regions of factor VIII transcripts provide potential mechanisms for the association. Low serum iron levels were also associated with venous thromboemboli (VTE): the age-adjusted $\mathrm{OR}$ of 0.91 (95\% $\mathrm{Cl} 0.86$ to 0.97$)$ per $1 \mu \mathrm{mol} /$ litre increase in serum iron implied a 2.5 -fold increase in VTE risk for a serum iron of $6 \mu \mathrm{mol} /$ litre compared with the mid-normal range $(17 \mu \mathrm{mol} /$ litre $)$. The association appeared to depend on factor VIII, as once adjusted for factor VIII, the association between VTE and iron was no longer evident.

Conclusions In this population, low serum iron levels attributed to inadequate replacement of haemorrhagic iron losses are associated with elevated plasma levels of coagulation factor VIII and venous thromboembolic risk. Potential implications for other clinical populations are discussed.

\section{INTRODUCTION}

Pulmonary emboli and deep venous thromboses cause major morbidity and mortality. ${ }^{1} 2$ The importance of venous thromboemboli (VTE) is emphasised by the Department of Health's mandatory VTE risk assessment data collection programme for NHS-funded acute care hospitals.

Patients with hereditary haemorrhagic telangiectasia $(\mathrm{HHT})^{3}$ represent a specific patient group

\section{Key messages}

What is the key question?

- Can we find new risk factors for venous thromboemboli (VTE) that might allow the development of a strategy to prevent pulmonary emboli and deep venous thromboses?

What is the bottom line?

- By focusing on the known VTE risk factor, coagulation factor VIII, low serum iron levels are identified as a biomarker for high factor VIII levels, and clinical VTE.

\section{Why read on?}

- Low serum iron levels are treatable by increasing iron intake, and thus represent a potentially reversible risk factor for pulmonary emboli and deep venous thromboses.

with unexplained high rates of VTE. ${ }^{4}$ Pulmonary emboli and anticoagulation carry particular hazards for these patients who exhibit sustained and chronic blood losses from nasal and gastrointestinal telangiectasia, and usually have arteriovenous malformations (AVMs) in pulmonary, hepatic and/or cerebral vascular beds. ${ }^{3}$ Elevated levels of coagulation factor VIII (FVIII) at least 6 months from any acute illness, infection or thrombosis are a strong predictor of long-term VTE risk in HHT. ${ }^{4}$ Similarly, elevated FVIII levels are a strong risk factor for VTE in the general population. ${ }^{5} 6$

In contrast to haemophilia A caused by mutations in the FVIII gene leading to severely reduced FVIII levels, to date, no unique genetic basis for elevated FVIII levels has been identified. ${ }^{7}$ In the CHARGE (Cohorts for Heart and Aging Research in Genomic Epidemiology) genome-wide association study of 23608 people of European descent, all five FVIII-associated loci were also associated with higher levels of von Willebrand factor (vWF), ${ }^{7}$ the glycoprotein with which FVIII circulates in a noncovalent complex. General population studies have delineated environmental factors that elevate plasma FVIII, and these parallel clinical risk factors 
for VTE. ${ }^{1}$ Thus FVIII levels are higher with increased age, ${ }^{8}$ and are acutely elevated in the setting of an acute phase inflammatory response. ${ }^{9}$

Patients with HHT provide a good group to further study the association between high FVIII and VTE because inflammation is not a prominent disease feature, yet patients display high rates of thrombotic events at relatively young ages. ${ }^{4}$ To identify novel biomarkers associated with elevated FVIII, stringently phenotyped HHT populations were examined. We hypothesised that this might facilitate a better understanding of why FVIII is elevated in patients with HHT, test the causal chain of biomarker-high FVIII-VTE, and importantly, allow the development of a strategy to reduce FVIII levels, and thereby also prevent pulmonary emboli and deep venous thromboses.

\section{METHODS}

The online supplement provides full details of patient assessments, power calculations and statistical methods.

\section{Ethical approvals}

A case notes review of patients with hereditary HHT was ethically approved by the Hammersmith, Queen Charlotte's, Chelsea, and Acton Hospital Research Ethics Committee (LREC 00/5792), and the approval remains valid. The study is also registered on the National Clinical Trials Database as NCT00230685 (PI Shovlin).

\section{STUDY PARTICIPANTS}

Patients were reviewed between 1 May 1999 and 7 January 2011 at the Hammersmith Hospital HHT/pulmonary AVM service in London, UK, a centre that receives nationwide referrals for these conditions. The sole eligibility criterion for this study was a definite diagnosis of HHT, assigned in the presence of at least three of four recognised international criteria of nosebleeds, mucocutaneous telangiectasia, visceral involvement and family history. ${ }^{10}$ Series 1 consisted of the 309 consecutive patients with HHT reviewed between 1999 and 2006. ${ }^{4}$ Series 2 ran from 2006 to 2011 , consisting solely of all $(n=300)$ patients with definite HHT who had not been part of series 1. Data are reported on all patients.

Patient histories recorded the presence or absence of HHTrelated symptoms and complications, other medical pathologies, and all treatments received. Routine assessments included a complete blood count; coagulation screen with fibrinogen; and biochemical screens of electrolytes, liver function, $\mathrm{C}$ reactive protein (CRP), and iron status (serum iron and transferrin saturation index (TfSI), with ferritin measured routinely from 2006 after iron associations emerged in series 1 analyses (Kulinskaya and Shovlin, 2006, unpublished)). In 1999, the optimal timing for the measurement of iron levels had been considered carefully based on reported diurnal variation in iron levels, ${ }^{11}$ and requirements to manage iron deficiency anaemia which is common in the population due to chronic nasal and/or gastrointestinal blood loss from HHT telangiectasia. ${ }^{3}$ Due to clinic arrangements, it was not possible to take blood samples in the early morning as recommended, ${ }^{11}$ and blood tests were taken in the late afternoon until September 2008, when sampling switched to lunchtime due to a change in clinic structures (for significance, see online supplementary figure 1). FVIII:Ag was included in routine blood tests from 2002, but not if it was within 6 months of a known confounding state such as VTE, infection, embolisation, surgery or pregnancy. vWF was included from 2006, after elevated FVIII levels were identified in series $1 .^{4}$ All patients underwent a screen for pulmonary AVMs that included standardised measurements of oxygen saturation in the erect posture, and for patients with pulmonary AVM undergoing subsequent embolisation, mean pulmonary artery pressure, measured routinely at angiography. ${ }^{12}$ Pulmonary emboli and deep venous thromboses were included as VTE endpoints only if confirmed by Doppler ultrasound, CT pulmonary angiography, other contrast studies, or ventilationperfusion scanning resulting in mismatched perfusion defects not explained by the presence of pulmonary AVMs. 'Community-restricted VTE' were defined as any spontaneous deep venous thromboses or pulmonary emboli that were not related to current or recent (within 6 weeks) hospitalisation.

\section{Statistical methods}

The distribution of patient-specific variables was assessed using one-way tables and data plots using Stata statistical software, release 11 (Statacorp, 2009, College Station, TX, USA). Identified outliers (prothrombin time $>16 \mathrm{~s}$; CRP $>40 \mathrm{iu} / \mathrm{ml}$ ) were excluded.

The distribution of FVIII:Ag was skewed and normalised by logarithmic transformation (data not shown). Log-transformed FVIII (lnFVIII) was used as the dependent variable for multiple regression analyses as previously. ${ }^{4}$ Levels were compared with concurrent indices and other parameters of clinical status. For each series, automated and manual stepwise forward and backwards linear regression analyses were performed using Stata 11 (Statacorp, TX, USA).

Age-standardised VTE incidence rates were calculated by allocating VTE cases to the decade of life in which they occurred. Incidence rates in each decade were calculated using the total number of person years per decade provided by the cohorts, using (Stata 11). Incidence rates were compared with previously published rates for the general population, ${ }^{2}$ and graphed using an exponential growth programme (GraphPad Prism 5.00, San Diego, California, USA $\left.{ }^{13}\right)$. Relationships between VTE and other patient-specific variables were assessed in stepwise logistic regression analyses using Stata 11. Interim analyses used the FVIII dataset for all iron indices, but these often differed between the time of FVIII measurement and VTE. For the final analyses, separate serum iron and TfSI measurements closest to VTE (interval 6 weeks to 60 months, mean 19 months), were used.

\section{RESULTS}

\section{Details of populations}

As demonstrated in online supplementary table 1, there were broad similarities among the 309 patients in series 1 and the 300 patients in series 2, with average ages of 49 and 46 years; a female bias of $62.7 \%$ and $60.3 \%$; and two-thirds having pulmonary AVMs (67\% and $72 \%$ ). Approximately one-quarter of the patients in each series $(29.6 \%$ and $24.7 \%)$ were using iron tablets for iron deficiency anaemia resulting from heavy iron losses as a result of nosebleeds and gastrointestinal bleeding. Similar proportions in each series were iron deficient.

\section{An association between low serum iron and high plasma FVIII} that is independent of inflammation

As expected, univariate analyses demonstrated that InFVIII levels were higher in older patients, and in the setting of raised inflammatory markers (online supplementary table 2 and supplementary figure 2). Supplementary table 2 also demonstrates the expected association between higher levels of FVIII and raised levels of vWF. vWF, which was only routinely available in series 2, accounted for $15.7 \%$ of the variance in FVIII levels in that series $(p<0.0001$, data not shown). 
Surprisingly, lnFVIII levels were also higher in patients in whom serum iron or TfSI values were low (supplementary table 2). Regression plots for FVIII with these commonly used markers of iron deficiency/overload are provided in figure 1A,B. In contrast, there was no relationship between FVIII and serum ferritin, another commonly used serum marker of iron status (online supplementary table 2; figure $1 \mathrm{C}$ ). There was also no association between serum iron and vWF $\left(\mathrm{N}=213 ; \mathrm{r}^{2}=0.009, \mathrm{p}>0.17\right)$.

Both FVIII levels ${ }^{4}$ and the severity of HHT telangiectasia ${ }^{3}$ increase with age, but in both series, the inverse relationships between InFVIII and serum iron (or TfSI) remained after adjustment for age and/or vWF (table 1).

Inflammation results in low iron levels ${ }^{14}$ and is recognised to elevate FVIII. ${ }^{9}$ Although the multiple regression analyses did not suggest that the low iron-high FVIII association was due to accompanying inflammation, we addressed this further. In keeping with a predominantly haemorrhagic cause of iron deficiency in patients with HHT, CRP and fibrinogen exhibited only weak relationships with serum iron or TfSI (figure $1 \mathrm{D}, \mathrm{E}$ ). In contrast, serum iron and TfSI were closely correlated as expected (figure 1F). Somewhat surprisingly, there was little correlation between serum iron (or TfSI) with serum ferritin (figure 1G). Ferritin is an acute phase protein, but the lack of association with serum iron/TfSI was not explained by concurrent confounding inflammatory responses (figure 1H,I). We concluded that the observed association between low serum iron/TfSI and high FVIII was independent of inflammation, and that despite a lack of diurnal variability (online supplementary figure 1), ferritin was not a robust means to evaluate the physiological state associated with low serum iron/TfSI in these cohorts (online supplementary figure 3).

Alternative mechanisms for the association between low serum iron/TfSI and high FVIII were considered. In conditions of low intracellular iron, more avid binding of iron reactive proteins to iron response elements (IREs) in untranslated regions (UTRs) of RNA transcripts inhibit protein translation, or enhance mRNA stability according to $5^{\prime}$ and $3^{\prime}$ UTR position respectively. ${ }^{14} 15$ The FVIII protein is encoded by a 26 exon transcript, but in endothelial cells, the same gene locus also generates several shorter transcripts with alternate first exons and UTRs. ${ }^{16}$ Examining FVIII transcript sequences ${ }^{15}$ identified a 5'UTR IRE within the alternate first exon of alternate transcript variant 2 (NM 019863, exon 22B, nt 11$42)$, with a predicted free energy of $-8.5 \mathrm{kCal}$, and a $3^{\prime}$ IRE sequence within the final exon of transcripts 1 (NM_000132, full length), 2 and 3 . This pattern would be predicted to enhance FVIII full length transcript 1 production in the setting of iron deficiency.
A

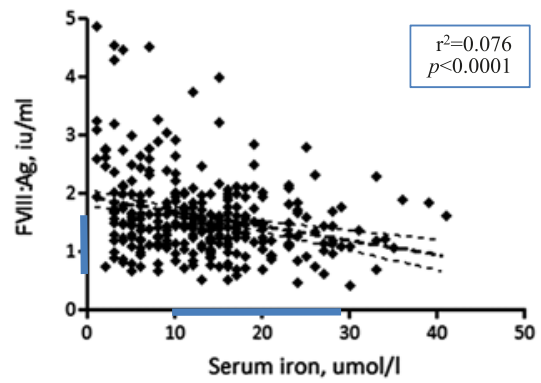

D

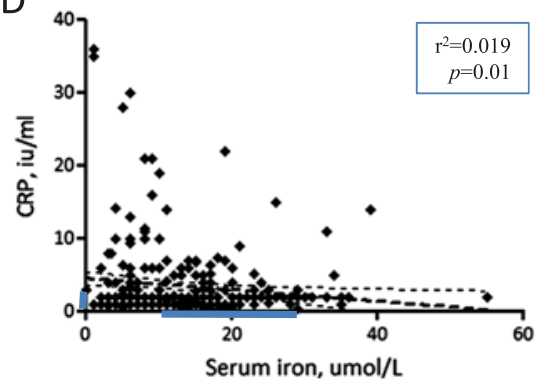

G

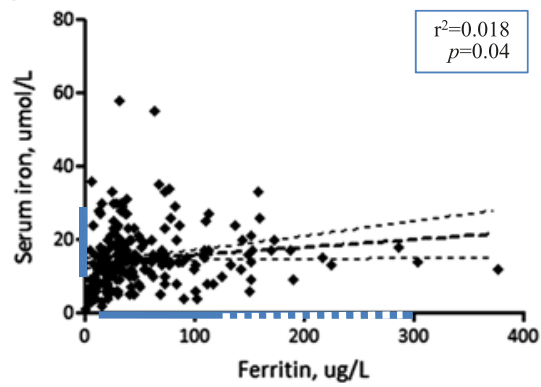

B

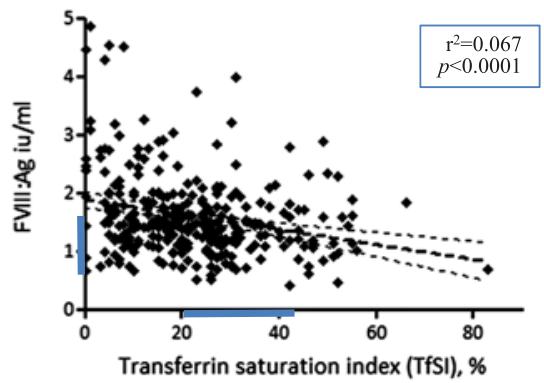

$E$

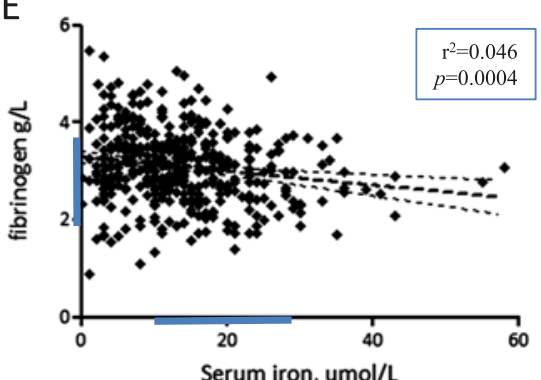

$\mathrm{H}$

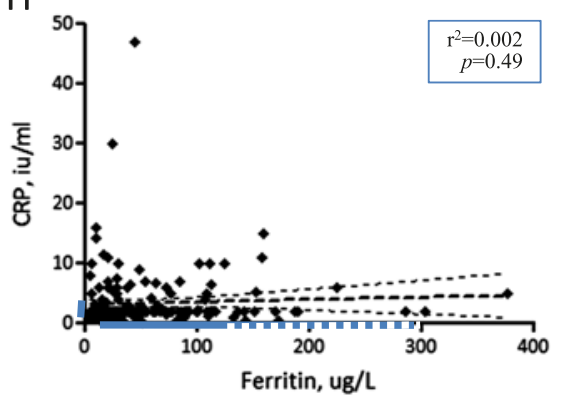

C
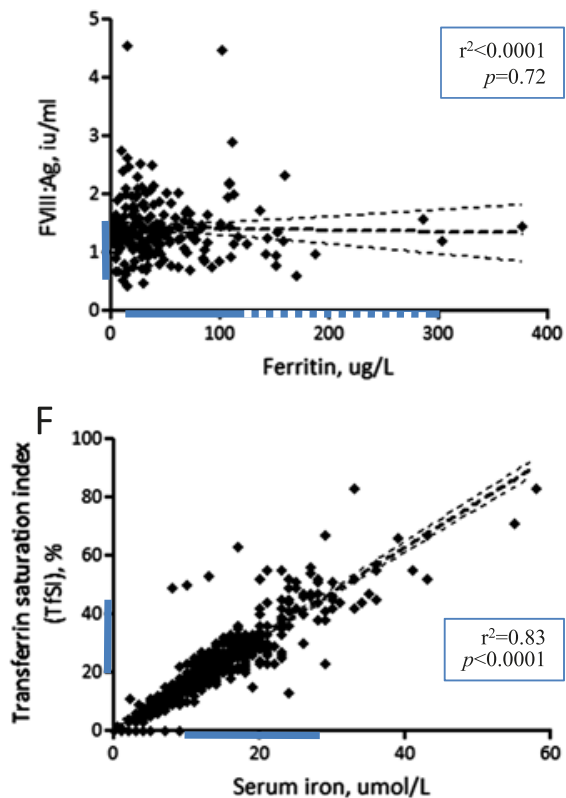

1

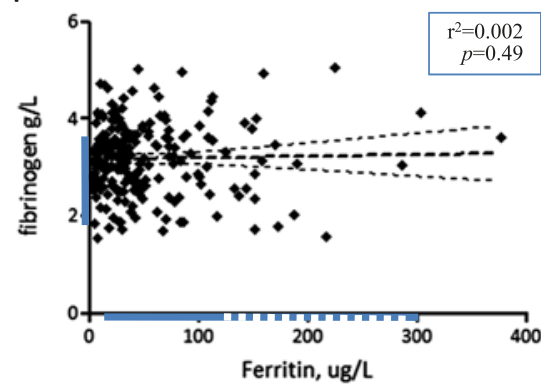

Figure 1 Factor VIII (FVIII) and associated regression plots. Scatter plots for key univariate associations in the combined series. The superimposed lines represent the linear regression line (bold) with $95 \%$ Cls. Boxes indicate the $r^{2}$ values and $p$ value for goodness of fit for each regression line. Thick bars on $x$ and $y$ axes indicate normal ranges, with dotted bars for extended ferritin normal range in men and post menopausal women. Upper panel: linear regression of FVIII with the iron indices of serum iron (A), transferrin saturation index (TfSI) (B) and ferritin (C). Middle panel: linear regression of serum iron with C-reactive protein (CRP) (D), fibrinogen (E), and TfSI (F). Bottom panel: linear regression of ferritin with serum iron (G), CRP (H), and fibrinogen (I). 
Table 1 Multiple regression of log-transformed factor VIII

\begin{tabular}{|c|c|c|c|}
\hline & $\begin{array}{l}\text { Regression } \\
\text { coefficient }\end{array}$ & $\mathbf{9 5 \%} \mathrm{Cl}$ & p value \\
\hline \multicolumn{4}{|l|}{ Series 1} \\
\hline Age & 0.0076 & 0.0026 to 0.013 & 0.003 \\
\hline Hypertension & 0.24 & 0.04 to 0.44 & 0.017 \\
\hline Serum iron & -0.0086 & -0.017 to 0.00033 & 0.059 \\
\hline \multicolumn{4}{|l|}{ Series 2} \\
\hline Von Willebrand factor & 0.37 & 0.27 to 0.46 & $<0.001$ \\
\hline Serum iron & -0.0092 & -0.015 to -0.0032 & 0.003 \\
\hline \multicolumn{4}{|c|}{$\begin{array}{l}\text { Multiple regression analyses for log-transformed factor VIII. For each model, the variables } \\
\text { identified as making a significant contribution to the final model, once adjusted for the } \\
\text { presence of other variables within the model, are presented. (Full model descriptive } \\
\text { parameters are presented in online supplementary table 3.) Note that von Willebrand factor } \\
\text { was only measured routinely for series 2, and therefore was not part of the series } 1 \text { model. } \\
\text { There was no significant difference if transferrin saturation index was used instead of serum } \\
\text { iron (data not shown). Higher order variables and interaction terms were not significant in } \\
\text { either model. To enhance statistical power, we considered pooling the series, but this was } \\
\text { not valid for these analyses, because in a combined stepwise regression model, the series } \\
\text { indicator was significant. }\end{array}$} \\
\hline
\end{tabular}

\section{VTE rates and HHT}

High proportions of patients with HHT are iron deficient (figure 1), and FVIII levels are known to be associated with VTE risk in HHT, as in the general population. Patients with HHT would therefore be predicted to have higher rates of VTE than the general population. Online supplementary table 4 provides details of individual pulmonary emboli and deep venous thrombotic events in the cohorts. The overall VTE incidence rate was 138.3 per 100000 patient years respectively. Age-standardised incidence rates were higher than for hospitalised patients

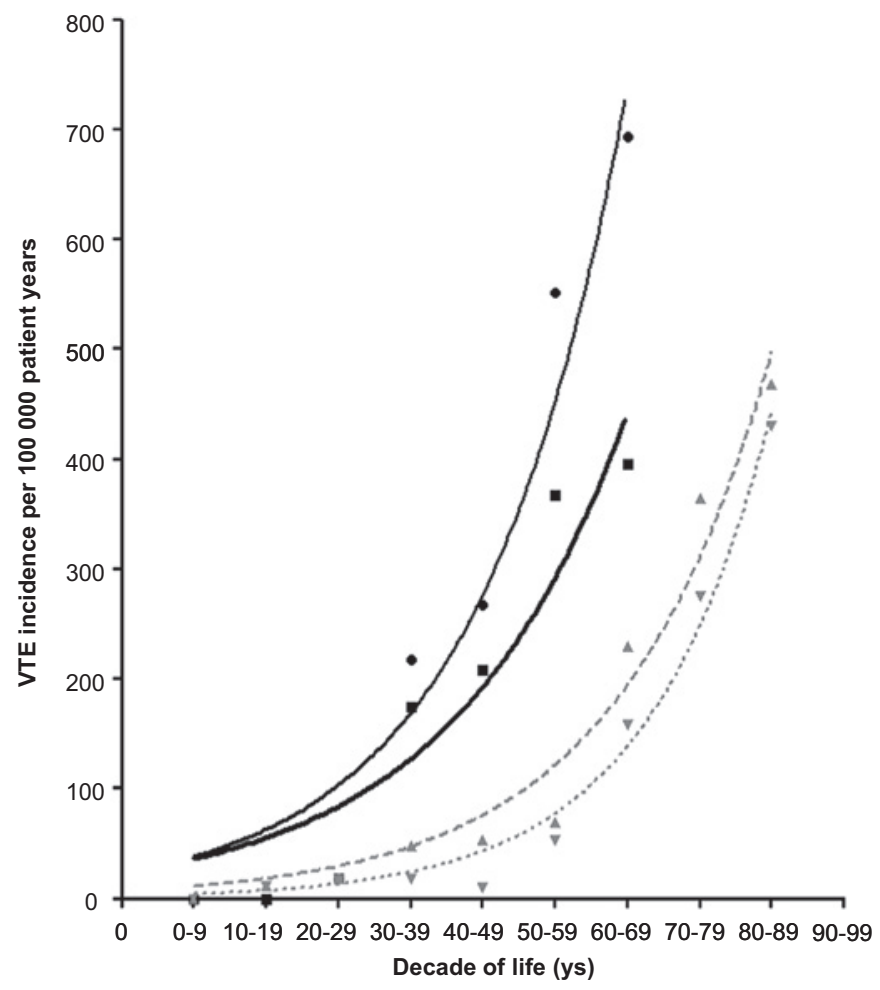

Figure 2 Comparison of age-specific venous thromboemboli (VTE) incidence rates for patients with haemorrhagic telangiectasia (HHT) and hospitalised patients from the general population. Circles, 1 pt line: all HHT VTE; squares, 2 pt line: 'community-restricted' HHT VTE. Grey symbols and lines from general population data: grey triangles, grey dashed line: hospitalised male patients ${ }^{2}$; grey dotted line: hospitalised female patients. ${ }^{3}$ from the general population (figure 2). The HHT data captured VTE occurring in patients currently or recently hospitalised with long-term inflammatory/immobility states, particularly following a pulmonary AVM-induced brain abscess. ${ }^{4}{ }^{17}$ However, age-standardised incidence rates for community-restricted VTE were also approximately twofold higher than rates for hospitalised patients from the general population (figure 2).

\section{An association between low serum iron and VTE risk that is independent of inflammation}

We examined whether low iron levels, or any other marker of HHT haemorrhage, were associated with VTE in the population. We separately analysed all VTE (which included inpatient events), and community-restricted VTE. As demonstrated in table 2 , in age, and age/inflammation-adjusted figures, only serum iron or serum TfSI were significantly associated with VTE risk. The age-adjusted OR for serum iron of 0.91 ( $95 \%$ CIs 0.86 to 0.97 for all VTE; 0.84 to 0.99 for community-restricted VTE) per $1 \mu \mathrm{mol} /$ litre increase in serum iron implied that a serum iron of $6 \mu \mathrm{mol} /$ litre would increase VTE risk approximately 2.5-fold compared with $17 \mu \mathrm{mol} /$ litre, the midpoint of the normal range. We concluded that the VTE association was with a status identified by low levels of serum iron/TfSI and not by other haemorrhage-related variables.

\section{The association between low serum iron and VTE depends on high FVIII}

To determine more complete VTE risk profiles, logistic regression analyses using all available patient variables were performed. For VTE events occurring in any setting ('All VTE'), and for VTE events only occurring in the community, FVIII levels were the strongest univariate predictor of VTE risk. Once adjusted for FVIII, neither iron/TfSI nor any other variable was significant at the $5 \%$ significance level in either setting (table 3 ).

\section{DISCUSSION}

Elevated plasma levels of FVIII are emerging as one of the strongest risk factors for VTE in the general population. ${ }^{5}{ }^{6}$ In health, genetic determinants of FVIII levels are primarily dependent on levels of its carrier protein vWF. ${ }^{7}$ The key findings from the current study are the identification of low serum iron levels as a potentially reversible biomarker for high FVIII levels and clinical VTE. These associations appear to operate independently to levels of vWF, or the inflammatory precipitants that are known to be associated with elevated FVIII and thromboembolic risk. Although the data were obtained in a specific patient group, they are supported by limited data from the general population literature that link iron deficiency or haemorrhage-associated anaemia with venous thromboses. ${ }^{18-20}$

The major strength of our study was the consistent timing of blood samples to late afternoon or lunchtime, capturing the time of daytime 'peaks' of serum iron and TfSI (online supplementary figure 1). This is important because the temporal variation of serum iron is complex, ${ }^{21} 22$ which makes interpretation of serum iron levels more difficult than is generally assumed. Serum ferritin levels are often considered a better marker of iron deficiency, but these too are difficult to interpret in the presence of coexisting pathologies ${ }^{23}$ : in the current study, ferritin values appeared to be disproportionately high in patients with severe hepatic AVM disease and iron deficiency, or those requiring weekly transfusions/iron infusions (online supplementary figure 3). The replicate HHT cohorts were statistically powerful due to the high prevalence of iron deficiency and high VTE rates. Additional strengths were the homogeneous populations, limited number of 
Table 2 ORs for associations between venous thromboemboli (VTE) and haemorrhage-associated variables

\begin{tabular}{|c|c|c|c|c|}
\hline & $\mathbf{N}$ & Age-adjusted OR & $\mathbf{N}^{\prime}$ & $\begin{array}{l}\text { Age and fibrinogen- } \\
\text { adjusted OR }\end{array}$ \\
\hline \multicolumn{5}{|l|}{ All VTE } \\
\hline Serum iron, at VTE & 493 & 0.91 (0.86 to 0.97$)$ & 449 & $0.90(0.84$ to 0.97$)$ \\
\hline Serum TfSI, at VTE & 494 & $0.95(0.92$ to 0.99$)$ & 450 & $0.95(0.91$ to 0.99$)$ \\
\hline Ferritin & 243 & $1.00(0.98$ to 1.01$)$ & 226 & 0.99 (0.98 to 1.01$)$ \\
\hline Haemoglobin & 543 & 0.94 (0.81 to 1.05$)$ & 487 & $0.94(0.80$ to 1.11$)$ \\
\hline Platelets & 550 & $1.00(1.00$ to 1.01$)$ & 495 & $1.00(1.00$ to 1.01$)$ \\
\hline On iron treatment (oral) & 593 & $1.52(0.73$ to 3.20$)$ & 496 & $1.93(0.89$ to 4.20$)$ \\
\hline Ever transfused & 599 & $0.93(0.31$ to 2.84$)$ & 494 & $1.00(0.32$ to 3.14$)$ \\
\hline Using hormones & 591 & $0.92(0.31$ to 2.70$)$ & 487 & $1.11(0.37$ to 3.34$)$ \\
\hline Using tranexamic acid & 592 & $1.02(1.00$ to 1.05$)$ & 489 & $1.30(0.27$ to 6.05$)$ \\
\hline \multicolumn{5}{|l|}{ Community restricted } \\
\hline Serum iron, at VTE & 493 & $0.91(0.84$ to 0.99$)$ & 449 & $0.90(0.82$ to 0.99$)$ \\
\hline Serum TfSI, at VTE & 494 & $0.95(0.91$ to 0.996$)$ & 450 & $0.94(0.89$ to 0.99$)$ \\
\hline Ferritin & 243 & $1.00(0.98$ to 1.01$)$ & 226 & $1.00(0.98$ to 1.01$)$ \\
\hline Haemoglobin & 543 & $0.89(0.72$ to 1.09$)$ & 487 & $0.89(0.72$ to 1.10$)$ \\
\hline Platelets & 550 & $1.00(1.00$ to 1.01$)$ & 495 & $1.00(0.99$ to 1.01$)$ \\
\hline On iron treatment (oral) & 599 & 0.90 (0.32 to 2.490$)$ & 496 & $1.07(0.37$ to 3.10$)$ \\
\hline Ever transfused & 599 & $0.94(0.20$ to 4.35$)$ & 494 & $1.00(0.21$ to 4.74$)$ \\
\hline Using hormones & 591 & $1.80(0.58$ to 5.54$)$ & 487 & $1.55(0.43$ to 5.64$)$ \\
\hline Using tranexamic acid & 593 & $0.94(0.12$ to 7.40$)$ & 489 & $1.25(0.15$ to 10.23$)$ \\
\hline
\end{tabular}

ORs for all VTE occurring in series 1 and 2 combined, with ORs significantly different to 1.00 denoted in bold. A series indicator variable was used to confirm the validity of series pooling. $\mathrm{N}$, number of observations for age-adjusted figures; $\mathrm{N}^{\prime}$, number including fibrinogen, selected as acute phase markers because available in 449 patients compared with 320 for C-reactive protein. Intravenous iron could not be part of any model due to the low frequency of use. TfSI, transferrin saturation index.

confounding diseases and immediate relevance to HHT. Furthermore, in many patients in the series, a polycythaemic stimulus due to pulmonary AVM-induced hypoxaemia masked the fall in haemoglobin due to iron deficiency. ${ }^{24}$ This poses difficult issues in HHT management, but importantly for this article, allowed iron deficiency to be distinguished from low haemoglobin/anaemia which would be more difficult in the general population. These factors may help explain why the iron deficiency-VTE associations have not been identified clearly in large general population epidemiological studies.

There are clear rationales why prothrombotic disease endpoints may be affected by iron deficiency, as evolutionary fitness would be enhanced by the capacity to augment coagulation (to limit blood loss at sites of vascular injury) when iron stores are depleted and the capacity to restore circulating blood haemoglobin is impaired. When iron deficiency prothrombotic risks have been reported previously, speculative mechanistic comments have focused on high platelet counts or inflammation. ${ }^{18-20}$ Our data did not identify such associations. The assumption that iron deficiency is associated with increased platelet counts has also been challenged elsewhere. ${ }^{25}$ The current study points to an alternative mechanism by which iron

Table 3 Logistic regression of venous thromboemboli (VTE)

\begin{tabular}{|c|c|c|}
\hline & OR (95\% Cl) & p value \\
\hline \multicolumn{3}{|l|}{ All VTE } \\
\hline FVIII & 3.09 (1.95 to 4.90$)$ & $<0.001$ \\
\hline \multicolumn{3}{|c|}{ Community VTE } \\
\hline FVIII & 2.88 (1.71 to 4.83 ) & $<0.001$ \\
\hline
\end{tabular}

Relationships between the binary dependent outcome variable of VTE with other patientspecific variables assessed by logistic regression analyses for all VTE (pseudo $r^{2}$ for final model $0.13, p<0.0001$ ) and community-restricted VTE (pseudo $r^{2}$ for final model 0.12 , $p=0.0002$ ). Stepwise regression based on the likelihood-ratio method was used to construct the models. Note that in each setting, factor VIII (FVIII) emerged as most significant in the first step, and no other variable was significant, once adjusted for FVIII levels in the 343 patients. In contrast to the FVIII regression analyses, in neither model was the series indicator variable significant, confirming the validity of pooling the series to enhance statistical power. deficiency may promote thromboses via elevation of plasma levels of coagulation factor FVIII. Mechanisms governing the regulation of plasma FVIII levels are not well understood, and so it is relevant that bioinformatic searches ${ }^{15}$ of endothelial FVIII transcripts ${ }^{16}$ predict the presence of IREs that suggest FVIII full length transcript 1 production may be enhanced in the setting of iron deficiency. We can therefore propose a plausible mechanism linking low serum iron to elevated FVIII.

Associations cannot however indicate direction or causality, particularly when these include potentially codependent variables. Thus we cannot rule out the possibility that high plasma FVIII levels (with accompanying VTE risk) somehow lower circulating levels of iron, although it is difficult to postulate a potential mechanism. Similarly, we cannot exclude chronic haemorrhage, or some component of HHT vascular pathology, causing both low serum iron and high FVIII. This has theoretical attractions because the transforming growth factor $\beta$ signalling pathways perturbed in $\mathrm{HHT}^{3}$ are related to pathways involved in hepcidin and iron regulation ${ }^{14}$ However, no associations of FVIII with other parameters of HHT haemorrhage were identified, nor was there evidence of iron deficiency in patients without major blood losses due to nosebleeds or gastrointestinal bleeding. Thus we predict that any contribution to the serum iron-FVIII relationship due to HHT-specific vascular pathology will be at best modest.

The crucial clinical question is whether low serum iron levels are provoking a prothrombotic state that could be reversed.

For patients with HHT, it is important to recognise that, as in the general population, provision of iron supplements can correct low serum iron levels in the face of ongoing blood loss. As illustrated by recent HHT guidelines, ${ }^{26}$ current practice is generally not to seek and treat iron deficiency, but instead to focus on identification and treatment of iron deficiency anaemia. The data within this manuscript would support the additional use of earlier corrective interventions before anaemia develops. 
Case studies and small comparative series link iron deficiency or haemorrhage-associated anaemia with venous thromboses in the general population, though FVIII levels were not reported. ${ }^{18-20}$ Management of iron deficiency anaemia is integral to virtually all medical and surgical disciplines including obstetric medicine where pulmonary emboli remain a relatively common cause of maternal mortality. ${ }^{27}$ Iron deficiency anaemia is estimated to affect at least 1 billion people worldwide, ${ }^{28}$ and is treatable by increasing iron intake to exceed total body losses. ${ }^{29}$ Thus the question of whether iron deficiency provides a potentially reversible prothrombotic stimulus is of wide general relevance.

There are also specific pulmonary vasculature implications, not least because pulmonary artery and microvascular endothelial cells synthesise and secrete FVIII. ${ }^{16} 3031$ Elevated plasma levels of FVIII are unusual among general thrombotic risk factors, as they are not only a risk factor for VTE, but are also associated with chronic thromboembolic and pulmonary arterial hypertension, for which pathology includes intrapulmonary microvascular thromboses. ${ }^{32} 33$ A prothrombotic potential for the recently observed unexplained iron deficiency in patients with pulmonary arterial hypertension ${ }^{34-36}$ is therefore intriguing.

In summary, data from replicate cohorts of patients with hereditary haemorrhagic telangiectasia strongly link low serum iron levels to VTE, with excess risk attributable to elevation of plasma levels of the prothrombotic coagulation factor FVIII. Further mechanistic and clinical examination in general population studies is warranted.

Acknowledgements CLS thanks Dr Joseph Eliahoo for additional advice on the use of Stata.

Funding This study received support from the NIHR Biomedical Research Centre Funding Scheme (MAL, CLS), the National Heart and Lung Institute's Undergraduate Teaching Funds (JAL), and donations from families and friends of British HHT patients. The study sponsors played no part in study design, in the collection, analysis and interpretation of data, in the writing of the report, or in the decision to submit the paper for publication.

\section{Competing interests None.}

Ethics approval Hammersmith, Queen Charlotte's, Chelsea, and Acton Hospital Research Ethics Committee.

Contributors JAL generated the series 2 database to mid 2010; performed interim statistical analyses using Stata, performed the SIRES searches; and wrote the first manuscript draft. RAM and MAL made all factor VIII (FVIII) measurements, and advised on thrombotic and FVIII concepts. JM advised on iron measurements, and measured iron indices in the patients and in the diurnal study. JEJ reviewed patients with pulmonary arteriovenous malformations, and performed all angiography with associated measurements including pulmonary artery pressure. EK performed initial statistical analysis of series 1; advised on final statistical methodology; and contributed to final statistical interpretation. CLS designed the study including diurnal assessments; reviewed the patients; generated the series 1 database; validated and extended the series 2 database; performed all statistical analyses presented; generated the figures; and wrote the final manuscript. All authors contributed to manuscript review, and approved the final version.

Provenance and peer review Not commissioned; externally peer reviewed.

\section{REFERENCES}

1. Kyrle PA, Rosendaal FR, Eichinger S. Risk assessment for recurrent venous thrombosis. Lancet 2010;376:2032-9.

2. Anderson FA Jr, Wheeler HB, Goldberg RJ, et al. A population-based perspective of the hospital incidence and case-fatality rates of deep vein thrombosis and pulmonary embolism. The Worcester DVT Study. Arch Intern Med 1991;151:933-8.

3. Shovlin CL. Hereditary haemorrhagic telangiectasia: pathogenesis, diagnosis and treatment. Blood Rev 2010;24:203-19.

4. Shovlin CL, Sulaiman NL, Govani FS, et al. Elevated factor VIII in hereditary haemorrhagic telangiectasia $(\mathrm{HHT})$ : association with venous thromboembolism. Thromb Haemost 2007;98:1031-9.

5. Kraaijenhagen RA, in't Anker PS, Koopman MM, et al. High plasma concentration of factor VIllc is a major risk factor for venous thromboembolism. Thromb Haemost 2000:83:5-9.
6. Kyrle PA, Minar E, Hirschl M. High plasma levels of FVIII and the risk of recurrent venous thromboembolism. N Engl J Med 2000;343:457-62

7. Smith NL, Chen MH, Dehghan A, et al. Novel associations of multiple genetic loci with plasma levels of factor VII, factor VIII, and von Willebrand factor: the CHARGE (Cohorts for Heart and Aging Research in Genome Epidemiology) Consortium. Circulation 2010;121:1382-92.

8. Luxembourg B, Schmitt J, Humpich $M$, et al. Intrinsic clotting factors in dependency of age, sex, body mass index, and oral contraceptives: definition and risk of elevated clotting factor levels. Blood Coagul Fibrinolysis 2009;20: 524-34

9. Begbie M, Notley C, Tinlin S, et al. The factor VIII acute phase response requires the participation of NFkappaB and C/EBP. Thromb Haemost 2000;84:216-22.

10. Shovlin CL, Guttmacher AE, Buscarini $E$, et al. Diagnostic criteria for hereditary hemorrhagic telangiectasia (Rendu-Osler-Weber syndrome). Am J Med Genet 2000:91:66-7

11. Burtis CA, Ashwood ER, Bruns DE, eds. Tietz Textbook of Clinical Chemistry and Molecular Diagnostics. 4th edn. St Louis: Elsevier, 2006.

12. Shovlin CL, Jackson JE, Bamford KB, et al. Primary determinants of ischaemic stroke/ brain abscess risks are independent of severity of pulmonary arteriovenous malformations in hereditary haemorrhagic telangiectasia. Thorax 2008;63:259-66

13. Motulsky HJ, Christopoulos A. Fitting models to biological data using linear and non linear regression. A practical guide to curve fitting. San Diego CA: GraphPad Software Inc., 2003. www.graphpad.com

14. Ganz T. Hepcidin and iron regulation, 10 years later. Blood 2011;117:4425-33.

15. Campillos $\mathbf{M}$, Cases I, Hentze MW, et al. SIREs: searching for iron-responsive elements. Nucleic Acids Res 2010;38(Suppl. W):360-7.

16. Shovlin CL, Angus G, Manning RA, et al. Endothelial cell processing and alternatively spliced transcripts of factor VIII. Potential implications for coagulation cascades and pulmonary hypertension. PLOS One 2010;5:e9154.

17. Shovlin CL, Wilmshurst $P$, Jackson JE. Pulmonary arteriovenous malformations and other pulmonary aspects of HHT. Eur Respir Mon 2011;54:218-45.

18. Stolz E, Valdueza JM, Grebe $\mathrm{M}$, et al. Anemia as a risk factor for cerebral venous thrombosis? An old hypothesis revisited. Results of a prospective study. J Neurol 2007;254:729-34.

19. Sundström A, Seaman $\mathrm{H}$, Kieler $\mathrm{H}$, et al. The risk of venous thromboembolism associated with the use of tranexamic acid and other drugs used to treat menorrhagia: a case-control study using the General Practice Research Database. BJOG 2009:116:91-7.

20. Adoh A, Kouame AN, Kouassi YF, et al. Etiological factors of deep vein thromboses in the limbs of Black African subjects. Med Trop (Mars) 1992;52:131-7.

21. Malhotra S, Garg SK, Khullar GK, et al. Kinetics of two different iron formulations and their effect on diurnal variation of serum iron levels. Methods Find Exp Clin Pharmacol 2004;26:417-20.

22. Hutchinson C, Conway RE, Bomford A, et al. Post-prandial iron absorption in humans: comparison between HFE genotypes and iron deficiency anaemia. Clin Nutr 2008;27:258-63

23. http://cks.nhs.uk/anaemia iron deficiency/management/scenario diagnosis/ investigations/interpreting_ferritin_levels (accessed 4 Nov 2011)

24. Roked F, Jackson JE, Fuld J, et al. Pulmonary thromboemboli modifying the natura history of pulmonary arteriovenous malformations. Am J Respir Crit Care Med 2011;183:828-9

25. Kuku I, Kaya E, Yologlu S, et al. Platelet counts in adults with iron deficiency anemia Platelets 2009;20:401-5.

26. Faughnan ME, Palda VA, Garcia-Tsao G, et al. International guidelines for the diagnosis and management of hereditary hemorrhagic telangiectasia. J Med Genet 2011:48:73-87.

27. Confidential enquiry into maternal deaths (CEMACH). Saving Mothers Lives 2003-2005 (Full Report). http://cemach.interface-test.com (accessed 29 Nov 2011).

28. McLean E, Cogswell M, Egli I, et al. Worldwide prevalence of anaemia, WHO Vitamin and Mineral Nutrition Information System, 1993-2005. Public Health Nutr 2009; 12:444-54

29. Centers for Disease Control and Prevention. Recommendations to prevent and control iron deficiency in the United States. MMWR Recomm Rep 1998:47:1-36.

30. Jacquemin $\mathbf{M}$, Neyrinck $A$, Hermanns MI, et al. FVIII production by human lung microvascular endothelial cells. Blood 2006;108:515-17.

31. Shahani T, Lavend'homme R, Luttun A, et al. Activation of human endothelial cells from specific vascular beds induces the release of a FVIII storage pool. Blood 2010;115:4902-9.

32. Bonderman D, Turecek PL, Jakowitsch J, et al. High prevalence of elevated clotting factor VIII in chronic thromboembolic pulmonary hypertension. Thromb Haemost 2003:90:372-6.

33. Wolf $\mathbf{M}$, Boyer-Neumann $\mathrm{C}$, Parent $\mathrm{F}$, et al. Thrombotic risk factors in pulmonary hypertension. Eur Respir J 2000:15:395-99.

34. Ruiter G, Lankhorst S, Boonstra A et al. Iron deficiency is common in idiopathic pulmonary arterial hypertension. Eur Respir J 2011;37:1386-91.

35. Soon E, Treacy CM, Toshner MR, et al. Unexplained iron deficiency in idiopathic and heritable pulmonary arterial hypertension. Thorax 2011;66:326-32.

36. Rhodes CJ, Howard LS, Busbridge M, et al. Iron deficiency and raised hepcidin in idiopathic pulmonary arterial hypertension: clinical prevalence, outcomes, and mechanistic insights. J Am Coll Cardiol 2011;58:300-9. 